\title{
CSCO BC guideline: updates for hormone receptor-positive breast cancer in $\mathbf{2 0 2 0}$
}

\author{
Fengrui $\mathrm{Xu}^{1,2}$, Zefei Jiang ${ }^{1}$ \\ ${ }^{1}$ Department of Breast Oncology, The Fifth Medical Center of Chinese PLA General Hospital, Beijing 100071, China; ${ }^{2}$ Department of Breast \\ Oncology, Academy of Military Medical Sciences, Beijing 100089, China \\ Correspondence to: Zefei Jiang. Department of Breast Oncology, The Fifth Medical Center of Chinese PLA General Hospital, No. 8 Dong Street, \\ Fengtai District, Beijing 100071, China. Email: jiangzefei@csco.org.cn.
}

Received: 28 February 2020; Accepted: 24 March 2020; Published: 10 April 2020.

doi: 10.21037/tbcr.2020.03.04

View this article at: http://dx.doi.org/10.21037/tbcr.2020.03.04

Breast cancer is the most common malignant tumor that threatens female life and health, more than half of which are hormone receptor (HR) positive breast cancer (1). In China, breast cancer ranks first in incidence and fifth in mortality among women. The relatively lower mortality rate is due to the disease characteristics and the development of standardized diagnosis and treatment (2). The guidelines of Chinese Society of Clinical Oncology Breast Cancer (CSCO BC) since 2017 were developed by Chinese scholars based on international standards and China's national conditions, providing a set of guidelines that are consistent with China's practice for breast cancer physicians in China.

The main basis for the update of CSCO BC guidelines is medical evidence, the availability of therapies, and new medical advances. With the successful research of innovative drugs such as CDK 4/6 inhibitors, PI3K-AKTmTOR inhibitors, and HDAC inhibitors, endocrine therapy has entered the era of targeted combination therapy $(3,4)$. Facing a variety of endocrine-targeted therapeutic drugs, determining the most viable treatment has become the main focus of clinicians. In the updated 2020 version of CSCO BC guideline, the panelists focused on more reasonable stratification of previous treatments, combined with the progress of endocrine therapy and changes in drug accessibility, to improve endocrine therapy under different strata. The main updates are as follows.

\section{Indications of endocrine therapy for advanced breast cancer}

Endocrine therapy has previously been considered suitable for HR-positive ABC (advanced breast cancer) that progresses slowly and benefits from previous endocrine therapy, while chemotherapy should be the prior choice for patients who progress fast or have visceral metastases. With the advent of endocrine-targeted drugs, the effectiveness of endocrine therapy has increased, patients' lifetime has also been prolonged, and endocrine therapy is becoming significantly more vital.

Recently, several studies on chemotherapy versus endocrine therapy reported preliminary results. The YongPEARL trial explored the differences of OFS (Ovarian function suppression) combined with Exemestane plus Palbociclib and Capecitabine in premenopausal ER positive ABC. The results showed that the endocrine therapy significantly prolonged PFS (progression-free survival) compared to chemotherapy. PEARL trial compared the efficacy of endocrine therapy combined with CDK 4/6 inhibitor to capecitabine in patients previous failing $\mathrm{AI}$ (aromatase inhibitor) treatment. The results showed that the endocrine-targeted therapy has equivalent effect and better tolerated. In addition, the results of the MonrchHER trial suggest that for triple-positive breast cancer, anti-HER2 targeted therapy combined with endocrine and CDK4/6 inhibitors has a better effect compared with chemotherapy plus anti-HER2 therapy.

Therefore, the panelists believe that, based on the advantages of endocrine-targeted therapy in improving survival and quality of life, the indications for endocrine therapy can be appropriately expanded. All patients with HR-positive advanced breast cancer (excluding patients with visceral crisis) should have access to endocrine combination 


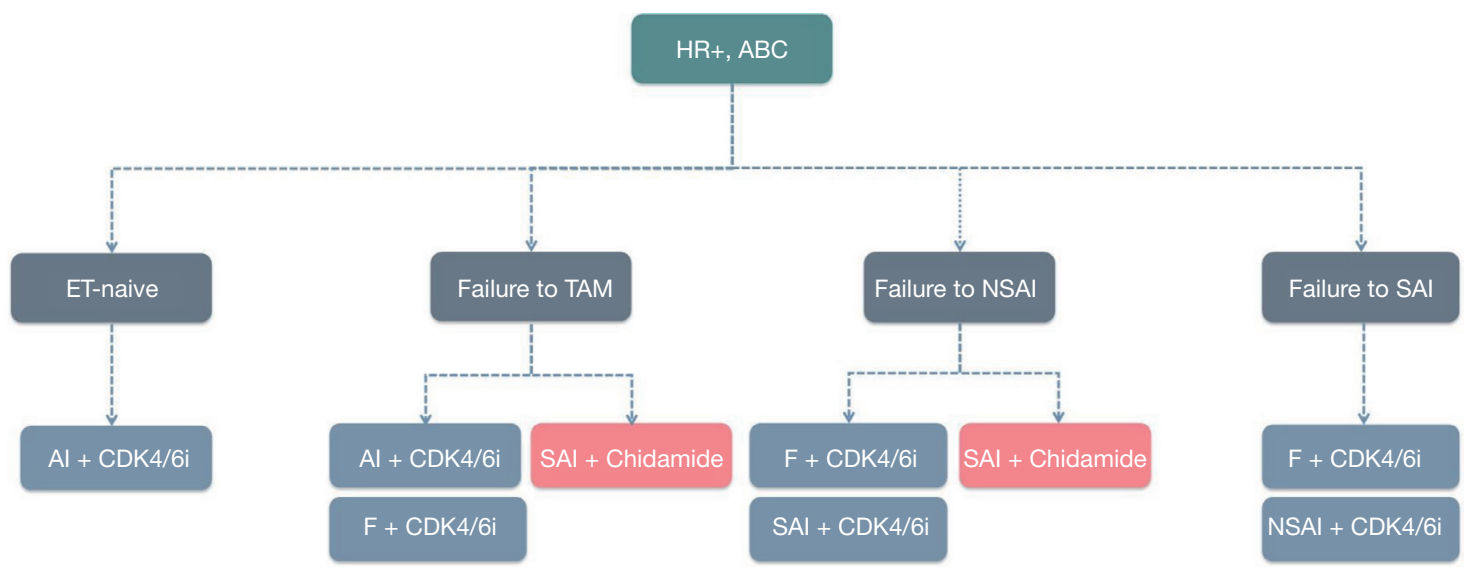

Figure 1 New strategies of for HR-positive advanced breast cancer. (I) Patients with endocrine therapy-naïve; (II) patients previously failed to TAM; (III) patients previously failed to NSAI; (IV) patients previously failed to SAI. HR, hormone receptor; ABC, advanced breast cancer; ET, endocrine therapy; F, fulvestrant; CDK 4/6i, CDK 4/6 inhibitors; NSAI, non-steroidal aromatase inhibitors; SAI, steroidal aromatase inhibitors.

targeted therapy.

\section{Recommendations for endocrine therapy of advanced breast cancer}

The increasing number of endocrine-targeted therapeutic drugs has provided new treatment opportunities for patients, but it has also increased the difficulty for clinicians in recommending treatment options. Both the NCCN guidelines and the St. Gallen breast cancer consensus identify the importance of endocrine-targeted therapy, but they also clearly state that "the optimal sequence of endocrine therapy has not been determined and depends on previous drug efficacy (neo-adjuvant/adjuvant and advanced), burden of disease, patient willingness, cost, and availability".

The CSCO BC guideline panel fully recognizes the value of endocrine-targeted therapy. Facing a variety of endocrine-targeted therapies, the panelists believe that CDK 4/6 inhibitors and HDAC inhibitors are the preferred treatments in China. For patients with endocrine therapynaive or previous failure to TAM, AI combined with CDK4/6 inhibitors or HDAC inhibitors are the preferred treatment. For AI-resistant patients, fulvestrant combined with CDK4/6 inhibitors or SAI combined with HDAC inhibitors can be recommended (Figure 1).

In the updated guideline, in order to better help clinicians determine the options for endocrine therapy, the panelists have stratified previous endocrine therapy cases. Different recommendation levels (I-III) were determined based on evidence, tolerance, and accessibility in each stratum. Overall, the 2020 version of the guideline emphasizes the importance of endocrine-targeted therapies (Table 1).

\section{Endocrine therapy-naive}

In the previous guidelines, considering the potency ratio, the expert panel listed AI plus CDK4/6 inhibitors as level II recommendations. This update raises this treatment to Level 1 recommendation due to three reasons: firstly, the results of MONALEESA-7 trial (5) showed that the addition of CDK 4/6 inhibitor can significantly improve the OS (overall survival) of patients. Secondly, the positive results reported by the MONARCHplus trial provided new evidence in Chinese patients. This study was a global multi-center clinical trial led by Chinese scholars. A total of 463 patients were divided into two cohorts. Cohort A was mainly endocrine therapy-naive patients, in which 306 patients were randomized to receive Abemaciclib combined with NSAI (non-steroidal aromatase inhibitors) or placebo combined with NSAI. The results showed that the endocrine-targeted therapy group can significantly prolong PFS compared to the 
Table 1 Recommendations for the endocrine therapy of HR-positive ABC in the 2020 version of CSCO breast cancer guideline

\begin{tabular}{|c|c|c|c|}
\hline Stratifications & Level 1 recommendations & Level 2 recommendations & Level 3 recommendations \\
\hline Failure to SAI & Fulvestrant + CDK4/6 inhibitor $(1 \mathrm{~A})$ & $\begin{array}{l}\text { (I) Fulvestrant (2A); } \\
\text { (II) NSAI + CDK4/6 inhibitor (2A) }\end{array}$ & $\begin{array}{l}\text { (I) NSAI (2B); } \\
\text { (II) TAM or Toremifene (2B); } \\
\text { (III) Progestin (2B) }\end{array}$ \\
\hline
\end{tabular}

HR, hormone receptor; NSAI, non-steroidal aromatase inhibitors; SAI, steroidal aromatase inhibitors; TAM, tamoxifen; HDAC, histone deacetylase.

endocrine mono-therapy group. This study enrolled a large number of Chinese patients and provided data on the efficacy and safety of CDK4/6 inhibitors in Chinese patients. Thirdly, CKD4/6 inhibitors are available in China market currently, and there is a substantial charitable donations policy.

\section{Failure to TAM}

The overall survival results of the second-line treatment studies MONALEESA-3 and MONARCH-2 have been published recently $(6,7)$. These studies included patients who had previously failed TAM treatment, and the results showed that the addition of CDK4/6 inhibitors could significantly improve overall survival. At the same time, based on accessibility concerns, the expert group generally agreed to adjust AI plus CDK4/6 inhibitors and fulvestrant plus CDK4/6 inhibitors to Level I recommendation.

It is worth noting that one of the most essential updates of the guideline is the inclusion of AI plus HDAC inhibitors in Level I recommendations. The evidence comes from the ACE trial (8). This study is the first phase III trial to show that epigenetic modulation plus endocrine therapy is a feasible and adequately tolerated strategy in patients with advanced, HR-positive, HER2-negative breast cancer that has progressed after previous endocrine therapy. Currently, chidamide is available in China, and based on the results of the ACE trial, its indication in breast cancer has been approved by CFDA. Therefore, the guideline panelists agreed that it is one of the most recommended treatments in such patients population.

\section{Failure to AI}

In the new version of $\mathrm{CSCO} \mathrm{BC}$ guideline, $\mathrm{AI}$ in previous treatments is further divided into NSAI and SAI, which is in consistent with clinical studies. The panelists believe that this change will bring more convenience to clinical operations.

For patients previously failed to NSAI: (I) this population is in accordance with one of the ACE trial enrollment groups. Based on the results of the study and considering the advantages of effectiveness and accessibility, the panelists recommended SAI plus HDAC inhibitors as a level I recommendation; (II) according to the results of PFS and OS in the PALOMA-3 (9), MONALEESA-3, MONARCH-2, and MONARCHplus studies, fulvestrant plus CDK4/6 inhibitors has also been recommended as level I, but the current economic burden is relatively heavier; (III) there is less data on the application of SAI plus CDK 4/6 inhibitors after NSAI failure, but based on its possible effectiveness, the panelists agreed to categorize it as a level II recommendation; (IV) BOLERO-2 trial (10) confirmed that exemestane combined with everolimus is one of the options, but everolimus has not yet obtained the breast cancer indication in China, so it is only recommended as level II.

For patients previously failed to SAI, the evidence for targeted endocrine therapy is mainly derived from CDK 
4/6 inhibitors. The PALOMA-3, MONALEESA-3, and MONARCH-2 studies all included patients who failed to SAI treatment. Therefore, the expert group agreed that fulvestrant plus CDK4/6 inhibitors could be used as a level I recommendation for this group of patients. Based on the potential effectiveness of CDK 4/6 inhibitors, the panel agreed to list NSAI plus CDK4/6 inhibitors as a level II recommendation. Meanwhile, the results of endocrine therapy combined with other targeted therapies such as HDAC inhibitors or PAM inhibitors are lacking in clinical studies and are not currently recommended for this population.

In conclusion, in these updates of endocrine therapy for HR-positive breast cancer, the panelists fully considered the research progress, which included both international research data and study evidence from China. Meanwhile, in combination with drug availability, tolerance, accessibility, etc., different levels of treatment recommendations are given to patients, helping oncologists to make more reasonable choices in clinical practice based on the specific situation of patients' treatment history.

\section{Acknowledgments}

Funding: None.

\section{Footnote}

Conflicts of Interest: Both authors have completed the ICMJE uniform disclosure form (available at http://dx.doi. org/10.21037/tbcr.2020.03.04). ZJ serves as an unpaid Editor-in-Chief of Translational Breast Cancer Research. FX has no conflicts of interest to declare.

Ethical Statement: The authors are accountable for all aspects of the work in ensuring that questions related to the accuracy or integrity of any part of the work are appropriately investigated and resolved.

Open Access Statement: This is an Open Access article distributed in accordance with the Creative Commons Attribution-NonCommercial-NoDerivs 4.0 International License (CC BY-NC-ND 4.0), which permits the noncommercial replication and distribution of the article with the strict proviso that no changes or edits are made and the original work is properly cited (including links to both the formal publication through the relevant DOI and the license). See: https://creativecommons.org/licenses/by-nc-nd/4.0/.

\section{References}

1. Lim E, Metzger-Filho O, Winer EP. The natural history of hormone receptor-positive breast cancer. Oncology (Williston Park) 2012;26:688-94, 696.

2. Chen W, Sun K, Zheng R, et al. Cancer incidence and mortality in China, 2014. Chin J Cancer Res 2018;30:1-12.

3. Finn RS, Martin M, Rugo HS, et al. Palbociclib and Letrozole in Advanced Breast Cancer. N Engl J Med 2016;375:1925-36.

4. Hortobagyi GN, Stemmer SM, Burris HA, et al. Updated results from MONALEESA-2, a phase III trial of firstline ribociclib plus letrozole versus placebo plus letrozole in hormone receptor-positive, HER2-negative advanced breast cancer. Ann Oncol 2018;29:1541-7.

5. Tripathy D, Im SA, Colleoni M, et al. Ribociclib plus endocrine therapy for premenopausal women with hormone-receptor-positive, advanced breast cancer (MONALEESA-7): a randomised phase 3 trial. Lancet Oncol 2018;19:904-15.

6. Slamon DJ, Neven P, Chia S, et al. Overall Survival with Ribociclib plus Fulvestrant in Advanced Breast Cancer. N Engl J Med 2020;382:514-24.

7. Sledge GW Jr, Toi M, Neven P, et al. The Effect of Abemaciclib Plus Fulvestrant on Overall Survival in Hormone Receptor-Positive, ERBB2-Negative Breast Cancer That Progressed on Endocrine TherapyMONARCH 2: A Randomized Clinical Trial. JAMA Oncol 2019. [Epub ahead of print],

8. Jiang $\mathrm{Z}, \mathrm{Li} \mathrm{W}, \mathrm{Hu} \mathrm{X}$, et al. Tucidinostat plus exemestane for postmenopausal patients with advanced, hormone receptor-positive breast cancer (ACE): a randomised, double-blind, placebo-controlled, phase 3 trial. Lancet Oncol 2019;20:806-15.

9. Turner NC, Slamon DJ, Ro J, et al. Overall Survival with Palbociclib and Fulvestrant in Advanced Breast Cancer. N Engl J Med 2018;379:1926-36.

10. Yardley DA, Noguchi S, Pritchard KI, et al. Everolimus plus exemestane in postmenopausal patients with $\mathrm{HR}(+)$ breast cancer: BOLERO-2 final progression-free survival analysis. Adv Ther 2013;30:870-84.

doi: $10.21037 /$ tbcr.2020.03.04

Cite this article as: $\mathrm{Xu} \mathrm{F}$, Jiang Z. CSCO BC guideline: updates for hormone receptor-positive breast cancer in 2020. Transl Breast Cancer Res 2020;1:3. 